\title{
INTERNALISING MODIFIED REALISABILITY IN CONSTRUCTIVE TYPE THEORY
}

\author{
ERIK PALMGREN
}

Department of Mathematics, Uppsala University

e-mail address: palmgren@math.uu.se

\begin{abstract}
A modified realisability interpretation of infinitary logic is formalised and proved sound in constructive type theory (CTT). The logic considered subsumes first order logic. The interpretation makes it possible to extract programs with simplified types and to incorporate and reason about them in CTT.
\end{abstract}

\section{Modified REALisabiLity}

Modified realisability interpretation is a well-known method for giving constructive interpretation of some intuitionistic logical system into a simple type structure [Tro73]. The method is used, for instance, in Minlog and Coq for extracting programs from proofs (cf. Sch04 and [Let04]). These programs are to a large extent free from the computationally irrelevant parts that might be present in programs arising from direct interpretations into constructive type theory. The realisability interpretation requires a separate proof of correctness, which is usually left unformalised.

In this note we present a completely formalised modified realisability interpretation carried out in the proof support system Agda Coq00. We shall here use what is called modified realisability with truth which has the property that anything realised is also true in the system (Theorem 1.2). One difference from usual interpretations as in Minlog is that the logic interpreted goes beyond first order logic: it is a (constructively) infinitary logic, which arises naturally from the type-theoretic notion of universe. Our extension to infinitary logic seems to be a novel result.

Agda is based on Martin-Löf constructive type theory [ML98, with an infinite hierarchy of universes $\# 0=$ Set, $\# 1=$ Type, $\# 2=$ Kind, $\# 3, \ldots$ Each of these universes is closed under the formation of generalised inductive data types. We define in Agda an inductive type SP of propositions, so called simple propositions, by induction: for each small type $A$ (i.e. a member of Set) an atomic proposition atom $(A)$ : SP is introduced; SP contains $\perp$ and is closed under propositional connectives $(\wedge, \vee, \rightarrow)$ and for any small type $A$ and any propositional function $P: A \rightarrow$ SP the quantified propositions $\forall(A, P)$ and $\exists(A, P)$ belong to SP. There is an obvious homomorphic embedding Tp of SP into the small types defined by $\operatorname{Tp}(\perp)=\emptyset, \operatorname{Tp}(\operatorname{atom}(A))=A, \operatorname{Tp}(P \vee Q)=\operatorname{Tp}(P)+\operatorname{Tp}(Q), \operatorname{Tp}(P \wedge Q)=$

2000 ACM Subject Classification: F.4.1.

Key words and phrases: Martin-Löf type theory, program extraction. 
$\operatorname{Tp}(P) \times \operatorname{Tp}(Q), \operatorname{Tp}(P \rightarrow Q)=\operatorname{Tp}(P) \rightarrow \operatorname{Tp}(Q), \operatorname{Tp}(\forall(A, P))=(\Pi x: A) \operatorname{Tp}(P(x))$ and $\operatorname{Tp}(\exists(A, P))=(\Sigma x: A) \operatorname{Tp}(P(x))$. We shall sometimes write $(\forall x: A) P(x)$ for $\forall(A, P)$ etc.

The simple propositions may be realised by terms from a simplified type structure. All atomic propositions will be realised by the unique element elt of the unit type Un. Define another homomorphism $\mathrm{Cr}$ (for crude type) from SP to small types by letting

$$
\begin{aligned}
\operatorname{Cr}(\perp) & =\mathrm{Un} \\
\operatorname{Cr}(\text { atom }(A)) & =\mathrm{Un} \\
\operatorname{Cr}(P \wedge Q) & =\operatorname{Cr}(P) \times \operatorname{Cr}(Q) \\
\operatorname{Cr}(P \vee Q) & =\operatorname{Cr}(P)+\operatorname{Cr}(Q) \\
\operatorname{Cr}(P \rightarrow Q) & =\operatorname{Cr}(P) \rightarrow \operatorname{Cr}(Q) \\
\operatorname{Cr}(\forall(A, P)) & =(\Pi x: A) \operatorname{Cr}(P(x)) \\
\operatorname{Cr}(\exists(A, P)) & =(\Sigma x: A) \operatorname{Cr}(P(x)) .
\end{aligned}
$$

The only difference from $\mathrm{Tp}$ is thus in the translation of absurdity and atoms. We note that a crude type may still be a dependent type, if the simple proposition is truly infinitary. For example, this is the case with $\operatorname{Cr}(\exists(A, P))$, if $A=N$ and $P(0)=\top, P(S(n))=Q(n) \wedge P(n)$.

Another variant of the crude type map $\mathrm{Cr}^{\prime}$ will be employed in Theorem 1.7 below, which is defined as $\mathrm{Cr}$, except that

$$
\mathrm{Cr}^{\prime}(\exists(A, P))=\mathrm{Un}+(\Sigma x: A) \mathrm{Cr}^{\prime}(P(x)) .
$$

The unit type appearing in the disjoint sum ensures that the type is never empty, which is crucial for interpreting the full absurdity axiom.

The modified realisability $\operatorname{MR}(S, r)$ of a simple proposition $S:$ SP by an element of crude type $r: \operatorname{Cr}(S)$ is defined as a small proposition (or small type) by the following recursion on $S$. (We use the identification of propositions and types for small types, so that $\wedge$ and $\vee$ are used interchangeably with $\times$ and + , respectively.)

$$
\begin{aligned}
\operatorname{MR}(\perp, r)= & \perp \\
\operatorname{MR}(\operatorname{atom}(P), r)= & P \\
\operatorname{MR}(A \wedge B, r)= & \operatorname{MR}(A, r .1) \wedge \operatorname{MR}(B, r .2) \\
\operatorname{MR}(A \vee B, \operatorname{inl}(s))= & \operatorname{MR}(A, s) \\
\operatorname{MR}(A \vee B, \operatorname{inr}(t))= & \operatorname{MR}(B, t) \\
\operatorname{MR}(A \rightarrow B, r)= & (\operatorname{Tp}(A) \rightarrow \operatorname{Tp}(B)) \\
& \wedge(\Pi s: \operatorname{Cr}(A))(\operatorname{MR}(A, s) \rightarrow \operatorname{MR}(B, r(s))) \\
\operatorname{MR}(\forall(A, P), r)= & (\Pi x: A) \operatorname{MR}(P(x), r(x)) \\
\operatorname{MR}(\exists(A, P), r)= & \operatorname{MR}(P(r .1), r .2) .
\end{aligned}
$$

Here $r .1$ and $r .2$ denote the first and second projections.

Remark 1.1. The above constructions work in many different type-theoretic settings. What is needed is a type universe $U$ closed under $\Pi, \Sigma,+$ and containing basic types 
Un and $\emptyset$. Moreover the inductive construction $\mathrm{SP}_{U}$ is should be made relative to $U$ instead of Set. Then

$$
\mathrm{Tp}_{U}: \mathrm{SP}_{U} \rightarrow U \quad \mathrm{Cr}_{U}: \mathrm{SP}_{U} \rightarrow U
$$

are defined by recursion on $\mathrm{SP}_{U}$ similarly to the above, and so is

$$
\mathrm{MR}_{U}:\left(\Pi s: \mathrm{SP}_{U}\right)\left(\mathrm{Cr}_{U}(s) \rightarrow U\right) \text {. }
$$

The following correctness, or conservativity, result states that each simple proposition, which is realised, is also true in the standard interpretation.

Theorem 1.2. For any $S: \mathrm{SP}$ and $r: \operatorname{Cr}(S)$, if $\operatorname{MR}(S, r)$ then $\operatorname{Tp}(S)$.

Proof. The proof goes by induction on $S$. For $S=\perp$ or $S=$ atom $(A)$ the result is immediate. For $S=A \rightarrow B$ we took care to define realisability so that this is direct as well. Here are two examples of the inductive step.

Suppose $\operatorname{MR}(A \vee B, r)$. If $r=\operatorname{inl}(s)$, then $\operatorname{MR}(A, s)$ is true. By the inductive hypothesis, we get $\operatorname{Tp}(A)$ and hence also $\operatorname{Tp}(A \vee B)$. The argument for $r=\operatorname{inr}(t)$ is similar.

Assume $\operatorname{MR}(\forall(A, P), r)$. Let $a \in A$. Then $\operatorname{MR}(P(a), r(a))$, and so by the inductive hypothesis $\operatorname{Tp}(P(a))$. Since $a$ was arbitrary we have actually $\operatorname{Tp}(\forall(A, P))$.

As a corollary there is an extraction theorem for $\forall \exists$-formulae:

Corollary 1.3. For small types $A$ and $B$ and a simple proposition $P(x, y)$ where $x: A$ and $y: B$, let

$$
S=(\forall x: A)(\exists y: B) P(x, y) .
$$

If $\operatorname{MR}(S, r)$ for some $r$, then there is some $f: A \rightarrow B$ such that $\operatorname{Tp}(P(x, f(x)))$ for all $x: A$.

Thereby the program $f$ extracted also satisfies its specification $\operatorname{Tp}(P(x, f(x)))$ within type theory. For $P(x, y)=$ atom $(R(x, y))$ this is equivalent to $R(x, f(x))$.

Remark 1.4. Note the difference in the $\forall$-case from usual interpretations, which go from theories to theories [Tro73]. It is not required that $\operatorname{Tp}(\Pi(A, P))$ is added to the condition, since this follows from the correctness theorem in the present internalised version.

We present an intuitionistic infinitary propositional logic $I P C_{\infty}^{-}$in type theory in which quantifiers are understood as infinitary versions of conjunction and disjunction. The system has a restriction on the absurdity axiom to atomic formulae.

$$
\begin{array}{cc}
A \vdash A & \frac{A \vdash B \quad B \vdash C}{A \vdash C} \\
A \vdash \operatorname{atom}(P), \text { for any inhabited } & P \\
A \wedge B \vdash A \quad A \wedge B \vdash B & \frac{C \vdash A \quad C \vdash B}{C \vdash A \wedge B} \\
\perp \vdash \operatorname{atom}(P) & \frac{A \vdash C \quad B \vdash C}{A \vee B \vdash C} \\
A \vdash A \vee B \quad B \vdash A \vee B & \frac{A \vdash B \rightarrow C}{A \wedge B \vdash C} \\
\frac{A \wedge B \vdash C}{A \vdash B \rightarrow C} & \frac{A \vdash \forall(S, P) \quad t: S}{A \vdash P(t)} \\
\frac{A \vdash P(t) \quad(t: S)}{A \vdash \forall(S, P)} & \frac{\exists(S, P) \vdash A \quad t: S}{P(t) \vdash A} \\
\frac{P(t) \vdash A \quad(t: S)}{\exists(S, P) \vdash A} &
\end{array}
$$


Remark 1.5. Note in particular that the existential quantifier is of the weak kind, as in first order logic. For $S=\emptyset$ each $\exists(S, P)$ works as absurdity constant. However, if we wish to avoid empty sets as types of realisers, the restricted absurdity axiom $\perp \vdash$ atom $(\mathrm{P})$ should be used. The full absurdity rule can be derived from the restricted one, for those propositions which do not include quantification over empty sets. By this procedure we can in principle extract simply typed programs as in Minlog.

We say that a sequent $A \vdash B$ is MR-realised, if there is some $r$ such that $\operatorname{MR}(A \rightarrow B, r)$ is true. A rule is realised if whenever all the sequents above the rule bar are realised, then so is the sequent below the bar.

Theorem 1.6. The axioms and rules of the system $I P C_{\infty}^{-}$are MR-realised.

To strengthen the weak absurdity axiom to the full axiom

$$
\perp \vdash A
$$

where $A$ : SP may be arbitrary, we use the crude type map $\mathrm{Cr}^{\prime}$ instead and introduce $\mathrm{MR}^{\prime}$. This is defined recursively as MR apart from the case for the existential quantifier:

$$
\begin{aligned}
& \operatorname{MR}^{\prime}(\exists(S, P), \operatorname{inl}(s))=\perp \\
& \operatorname{MR}^{\prime}(\exists(S, P), \operatorname{inr}(t))=\operatorname{MR}^{\prime}(P(t .1), t .2) .
\end{aligned}
$$

Theorem 1.2 and Corollary 1.3 now go through with $\mathrm{MR}^{\prime}$ and $\mathrm{Cr}^{\prime}$ in place of $\mathrm{MR}$ and Cr.

The proof of soundness of the logical rules and axioms is similar as for Theorem 1.6. with the exception for the verification of the absurdity rule, and the left existential rule. This requires a special device. Namely a function which to each $P$ : SP assigns an element, called element $(P)$, of $\mathrm{Cr}^{\prime}(P)$ is necessary. This function is defined straightforwardly by recursion on $P$. Some key clauses are

$$
\begin{aligned}
\text { element }(\exists(A, P)) & =\operatorname{inl}(\text { elt }) \\
\text { element }(\forall(A, P)) & =\lambda x \cdot \text { element }(P(x)) \\
\text { element }(A \vee B) & =\operatorname{inl}(\operatorname{element}(A)) .
\end{aligned}
$$

Observe that no such element need to exist when employing the first definition of $\mathrm{Cr}$, e.g. in the case $\operatorname{Cr}(\exists(\emptyset, P))=(\Sigma x: \emptyset) \operatorname{Cr}(P(x))$.

Theorem 1.7. The axioms and rules of the full system $I P C_{\infty}\left(I P C_{\infty}^{-}\right.$and the full absurdity axiom) are $\mathrm{MR}^{\prime}$-realised.

We mention some useful mathematical axioms that are realisable:

Lemma 1.8. For each propositional function $P: \mathbb{N} \rightarrow \mathrm{SP}$ the induction scheme

$$
P(0) \wedge(\forall x: \mathbb{N})[P(x) \rightarrow P(S(x))] \rightarrow(\forall x: \mathbb{N}) P(x)
$$

is both MR-realised and $\mathrm{MR}^{\prime}$-realised. 
Lemma 1.9. For any binary propositional function $P: A \times B \rightarrow \mathrm{SP}$ the type-theoretic choice principle

$$
(\forall x: A)(\exists y: B) P(x, y) \rightarrow(\exists g: A \rightarrow B)(\forall x: A) P(x, g(x))
$$

is MR-realisable. In case $B$ is inhabited, the principle is $\mathrm{MR}^{\prime}$-realisable as well.

Proof. The non-trivial part is to prove the second statement. Suppose $b_{0}: B$ and $r: \mathrm{Cr}^{\prime}(S)$ and $p: \mathrm{MR}^{\prime}(S, r)$, where $S=(\forall x: A)(\exists y: B) P(x, y)$. Define an auxiliary operation $f(x, w):(\Sigma y: B) \operatorname{Cr}^{\prime}(P(x, y))$ where $x: A$ and $w: \operatorname{Cr}^{\prime}((\exists y: B) P(x, y))$, by cases

$$
\begin{aligned}
& f(x, \operatorname{inl}(u))=\left\langle b_{0}, \text { element }\left(P\left(x, b_{0}\right)\right)\right\rangle \\
& f(x, \operatorname{inr}(y))=y .
\end{aligned}
$$

The realiser $k$ for the implication is now given by

$$
k(r)=\langle\lambda x . f(x, r(x)) .1, \lambda x . f(x, r(x)) .2\rangle
$$

To prove it is a realiser, use $\perp$-elimination for the case $r(x)=\operatorname{inl}(u)$.

The following result is often useful to verify realisability.

Lemma 1.10. If the Tp-translation of the proposition

$$
\left(\forall x_{1}: A_{1}\right) \cdots\left(\forall x_{n}: A_{n}\right)\left[Q\left(x_{1}, \ldots, x_{n}\right) \rightarrow P\left(x_{1}, \ldots, x_{n}\right)\right]
$$

is true and $P$ is atomic or $\perp$, then the proposition is MR-realised as well as $\mathrm{MR}^{\prime}$-realised.

Proof. The realising function is trivial for such a proposition: $\left(\lambda x_{1}\right) \cdots\left(\lambda x_{n}\right)(\lambda r)$ elt. Theorem [1.2 a special property of modified realisability with truth, is necessary here.

Many stronger "transfer principles" are possible to establish. See BBS02] for further results and references.

\section{An EXAMPLE}

We test the formalisation and extraction procedure on a simple example, which is due to Berger and Schwichtenberg. The extracted function computes Fibonacci numbers efficiently by "memoization."

A binary predicate $G$ on natural numbers is given. From the axioms

$(\mathrm{Ax} 1) G(0,0)$

$(\mathrm{Ax} 2) G(1,1)$

$(\mathrm{Ax} 3)(\forall m, k, \ell)[G(m, k) \wedge G(S(m), \ell) \rightarrow G(S(S(m)), k+\ell)]$.

one derives by induction and intuitionistic logic the proposition

(P) $(\forall x)(\exists k, \ell) G(x, k) \wedge G(S(x), \ell)$.

Thus there is some realiser $f$ so that

$$
\mathrm{MR}(\mathrm{Ax} 1 \& \mathrm{Ax} 2 \& \mathrm{Ax} 3 \vdash \mathrm{P}, f) .
$$

The extracted program $p$ (which is $\mathrm{fib}$ _prog in the Appendix) for computing the Fibonacci sequence is then given by

$$
p(x)=f(n c, x) \cdot 1
$$

where $n c$ (nocontent in the Appendix) is the trivial realiser for Ax1 \& Ax2 \& Ax3. After a normalisation process one gets the program: 


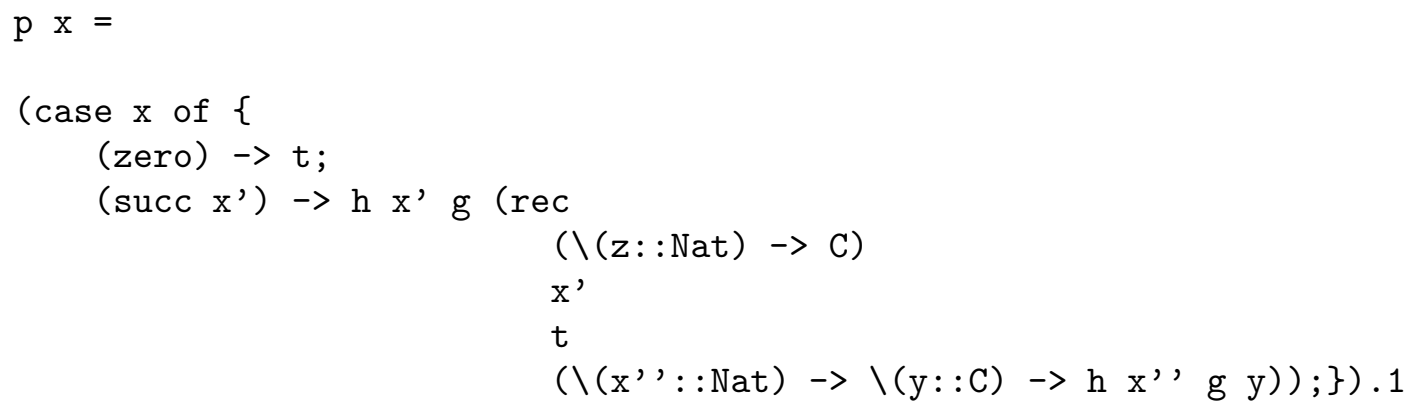

where

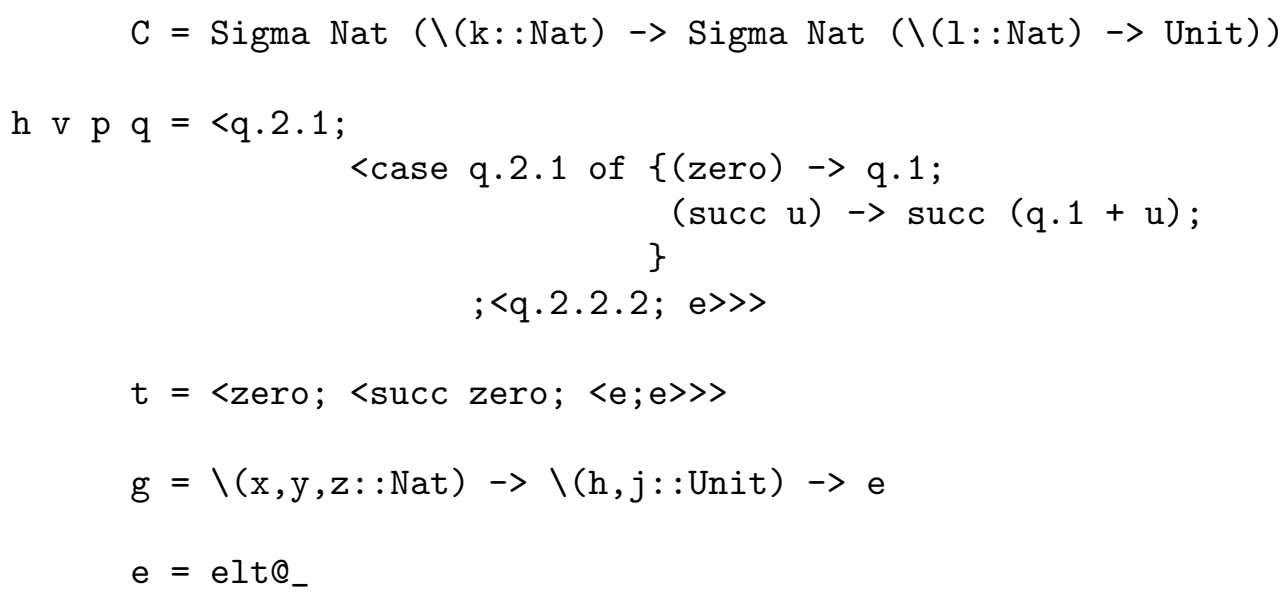

Remark 2.1. Note that all truly dependent types have disappeared. The type $C$ is really the type $\mathrm{N} \times(\mathrm{N} \times \mathrm{Un})$.

The normalised program has been computed using the partial normalisation procedure of Agda on selected subexpressions, and was thus not completely automatic. We also introduced the abbreviations $C, h, t, g, e$ by hand. Some syntactical sugar for lambda expressions and pairs is added.

\section{THE FORMALISATION}

The formalisation have been carried out in Agda/IAgda (version 2003-08-09) with the aid of the graphical user interface Alfa. The relevant files are available at the URL

www.math.uu.se/ palmgren/modif

\section{REFERENCES}

$\left[\mathrm{BBS}^{+}\right.$98] H. Benl, U. Berger, M. Seisenberger, H. Schwichtenberg, and W. Zuber, Proof theory at work: Program development in the Minlog system, Automated Deduction, Vol. II (W. Bibel and P.H. Schmitt, eds.), Kluwer, 1998.

[BBS02] U. Berger, W. Buchholz, and H. Schwichtenberg, Refined program extraction from classical proofs, Annals of Pure and Applied Logic 114 (2002), 3-25.

[Coq00] C. Coquand, The interactive theorem prover agda, Chalmers University of Technology, Department of Computer Science and Engineering, URL: www.cs.chalmers.se/ ${ }^{\sim}$ catarina/agda/, 2000. 
[Let04] P. Letouzey, Programmation fonctionelle certifiée: L'extraction de programmes dans l'assistant Coq., Ph.D. thesis, Université de Paris Sud, 2004.

[ML98] P. Martin-Löf, An intuitionistic theory of types, Twenty-Five Years of Type Theory (G. Sambin and J.M. Smith, eds.), Oxford University Press, 1998, pp. 127-172.

[Sch04] H. Schwichtenberg, Minimal logic for computable functions., Mathematisches Institut der Universität München, Preprint October, 2004.

[Tro73] A.S. Troelstra, Metamathematical investigation of intuitionistic analysis and arithmetic, Springer, 1973. 